\title{
Tetramethylpyrazine reduces inflammation in the livers of mice fed a high fat diet
}

\author{
BING CHEN ${ }^{1}$, YALUAN MA $^{1}, \mathrm{XIN} \mathrm{XUE}^{1}, \mathrm{JIE} \mathrm{WEI}^{2}, \mathrm{GANG} \mathrm{HU}^{2}$ and YAJUN LIN ${ }^{2}$ \\ ${ }^{1}$ Institute of Basic Theory for Chinese Medicine, China Academy of Chinese Medical Sciences, Beijing 100700; \\ ${ }^{2}$ Key Laboratory of Geriatrics, Beijing Hospital, National Center of Gerontology, Beijing 100730, P.R. China
}

Received July 11, 2018; Accepted January 14, 2019

DOI: $10.3892 / \mathrm{mmr} .2019 .9928$

\begin{abstract}
The present study aimed to assess the protective effects of tetramethylpyrazine (TMP) on the livers of mice fed a high fat diet. The mice were divided into five groups: Regular diet; high fat diet; simvastatin-treated; and low and high dose TMP-treated groups. The results demonstrated that, compared with the control group, serum glucose, total cholesterol (TC) and low-density lipoprotein cholesterol levels were increased in the model group. Additionally, compared with the model group, simvastatin lowered the TC level, whereas TMP did not. Compared with the control group, the level of malondialdehyde (MDA) in the liver tissue was increased and the level of glutathione peroxidase (GSH-pX) in the liver tissue was decreased in the model group. Furthermore, compared with the model group, TMP decreased the level of MDA and increased the level of GSH-Px; however, simvastatin did not have these effects. Immunohistochemistry and western blotting were performed; the results showed that, compared with the control group, the levels of inflammatory factors (tumor necrosis factor- $\alpha$ and interleukin-6) in the liver tissue were increased, and the ratio of phosphorylated (p)-nuclear factor $\kappa \mathrm{B}$ $(\mathrm{NF}-\kappa \mathrm{B}) / \mathrm{NF}-\kappa \mathrm{B}$ was also increased in the model group. The addition of TMP and simvastatin demonstrated that, compared with the model group, the inflammatory factor levels and the ratio of $\mathrm{p}-\mathrm{NF}-\kappa \mathrm{B} / \mathrm{NF}-\kappa \mathrm{B}$ were decreased. In addition, liver lipid deposition was examined in the model group using hematoxylin and eosin staining and Oil Red $\mathrm{O}$ staining, and the results showed that TMP and simvastatin reduced liver lipid deposition. Furthermore, compared with the control group, the reactive oxygen species (ROS) level in the liver tissue was increased. Compared with that in the model group, TMP and simvastatin decreased the ROS level. In conclusion, TMP, similar to simvastatin, exerted a notable hepatoprotective effect on mice fed a
\end{abstract}

Correspondence to: Professor Yajun Lin, Key Laboratory of Geriatrics, Beijing Hospital, National Center of Gerontology, 1 Dahua Street, Dongdan, Dongcheng, Beijing 100730, P.R. China E-mail: linyajun2000@126.com

Key words: tetramethylpyrazine, C57BL/J mice, inflammatory factors, non-alcoholic fatty liver, reactive oxygen species high fat diet with non-alcoholic fatty liver disease, by inhibiting inflammatory factors and the $\mathrm{p}-\mathrm{NF}-\kappa \mathrm{B} / \mathrm{ROS}$ signaling pathway.

\section{Introduction}

Non-alcoholic fatty liver disease (NAFLD) is a type of pathological fat accumulation in the liver and is a major health condition in the world (1). NAFLD includes a wide spectrum of conditions, ranging from non-alcoholic fatty liver (NAFL) to non-alcoholic steatohepatitis (NASH) (2,3). NAFL is characterized by an accumulation of hepatocellular lipids, mainly triglycerides, whereas NASH is identified by the addition of inflammation (4). It is estimated that $10-20 \%$ of patients with NAFL also develop NASH (5), and NASH is associated with a $>10$-fold increased risk $(2.8$ vs. $0.2 \%)$ of liver-associated mortality and twice the risk of cardiovascular disease (6). Therefore, the increasing prevalence of NASH and the increased risk of cardiovascular disease and liver-associated mortality have led to a demand for medical therapy. However, no pharmacological therapy has been shown to be effective for long-term use (7).

Tetramethylpyrazine (TMP) is a biologically active alkaloid extracted from Ephedra sinica (8), which has been widely used in Chinese herbal medicines for various purposes, including treating cardiovascular and cerebrovascular defects, and anti-oxidation, antifibrotic, anti-nociceptive, anti-inflammatory and anti-neoplastic activities $(9,10)$. TMP can decrease arsenic-induced reactive oxygen species (ROS) production, enhance glutathione peroxidase (GSH-pX) levels, prevent mitochondrial dysfunction, and suppress the activation of pro-inflammatory signals and the development of autophagy and apoptosis (11). However, the effect of TMP on the progression of NAFL to NASH remains to be fully elucidated. The present study observed the protective effect of TMP on NASH in mice, examined its therapeutic mechanism and provided a theoretical basis for its clinical application.

\section{Materials and methods}

The present study was approved by The Research Ethics Committee of the China Academy of Chinese Medical Sciences (Beijing, China), in accordance with the National Institutes of Health Guidelines for the Care and Use of Laboratory Animals (National Institutes of Health, Bethesda, MD, USA). All animals were treated in accordance with the guidelines and regulations 
for the use and care of animals of the Center for Laboratory Animal Care, China Academy of Chinese Medical Sciences.

Chemicals and reagents. TMP and simvastatin were purchased from Nanjing Jingzhu Bio-technology Co., Ltd. (Nanjing, China). An Oil Red O staining kit, 2',7'-dichlorofluorescin diacetate (DCFH-DA) and chloral hydrate were obtained from Sigma-Aldrich;Merck KGaA (Darmstadt,Germany). Superoxide dismutase (SOD), GSH-px and malondialdehyde (MDA) kits were purchased from Nanjing Jiancheng Bioengineering Institute (Nanjing, China). Antibodies against tumor necrosis factor- $\alpha$ (TNF- $\alpha$, cat. no. 3707), interleukin-6 (IL-6, cat. no. 12912), nuclear factor- $\kappa \mathrm{B}$ (NF- $\kappa \mathrm{B}$, cat. no. 6956), and phosphorylated (p)-NF-kB (cat. no. 13346) were acquired from Cell Signaling Technology, Inc. (Danvers, MA, USA). Secondary antibodies against rabbit (cat. no. 7074) and mouse (cat. no. 7076) immunoglobulin G were obtained from Cell Signaling Technology, Inc. An antibody against $\beta$-actin (cat. no. sc-81178) was acquired from Santa Cruz Biotechnology, Inc. (Dallas, TX, USA).

Animals and establishmentof an NAFLmodel.The C57BL/J mice ( $\mathrm{n}=60$, male to female ratio 1:1) were purchased from Beijing HFK Bioscience Co., Ltd. (Beijing, China). These mice (aged 8 weeks old, body weight 22-24 g) were bred in a specific pathogen-free laboratory (temperature, $20-24^{\circ} \mathrm{C}$; humidity, $40-70 \%$ ) at the China Academy of Chinese Medical Sciences Laboratory Animal Center (Beijing, China). The mice were acclimated to the feed for 1 week prior to the initiation of experimental intervention. In the present study, the murine NAFLD model was established by feeding mice a high fat diet for 8 weeks. A proportion of the mice $(n=12)$ were fed a regular diet, whereas others $(n=48)$ were fed a high fat diet (60 kcal \% fat; cat. no. D12492; Research Diets, Xietong Organism Co., Ltd., Nanjing, China). The standard used for establishment of the murine model for NAFLD was the NAFLD activity score. Food and water were provided ad libitum throughout the study. After 8 weeks, the mice fed a high fat diet were divided into four groups: Model group $(\mathrm{n}=12)$; $4 \mathrm{mg} / \mathrm{kg} /$ day simvastatin-treated group $(\mathrm{n}=12)$; and $100(\mathrm{n}=12)$ and $200(n=12) \mathrm{mg} / \mathrm{kg} /$ day TMP-treated groups. From 9 weeks, the mice received either distilled water (control and model groups), $4 \mathrm{mg} / \mathrm{kg} /$ day simvastatin (simvastatin-treated group), or 100 or $200 \mathrm{mg} / \mathrm{kg} /$ day TMP (TMP-treated groups) by forced oral ingestion. On day 85 , after $12 \mathrm{~h}$ of fasting, the mice were anesthetized with chloral hydrate $(500 \mathrm{mg} / \mathrm{kg})$ by intraperitoneal injection and blood samples were taken from the inferior vena cava. The survival of mice was confirmed by monitoring their breathing and heartbeat. Subsequently, the mice were sacrificed by cervical dislocation and their livers were quickly removed.

Hematological examination. In order to detect the protective effects of TMP on the liver, serum was collected. The serum levels of glucose, alanine aminotransferase (ALT), aspartate aminotransferase, total bilirubin, triglyceride (TG), total cholesterol (TC), low-density lipoprotein-cholesterol (LDL-C) and high-density lipoprotein-cholesterol were measured using standard protocols in the clinical laboratory.

Histology and immunohistochemistry. For histological analysis, liver tissues fixed with $4 \%$ buffered paraformaldehyde were embedded in paraffin, and $3-\mu \mathrm{m}$-thick sections were prepared. The sections were then stained with hematoxylin and eosin. The sections of liver tissues were evaluated using a light microscope (magnification, x200; Olympus Corporation, Tokyo, Japan) for the degree of steatosis (low to medium-power evaluation of parenchymal involvement by steatosis $<5 \%$, score $0 ; 5-33 \%$, score $1 ;>33-66 \%$, score 2 ; $>66 \%$ score 3 ), lobular inflammation (overall assessment of all inflammatory foci, no foci, score $=0$; $<2$ foci per $\times 200$ magnification field, score 1; 2-4 foci per x200 magnification field, score 2; >4 foci per x200 magnification field, score 3) and hepatocellular ballooning (none, score 0; few balloon cells, score 1; numerous cells/prominent ballooning, score 2) by an experienced pathologist blinded to the clinical data, according to Kleiner et al (12). The unweighted sum of these three variables was used to calculate the NAFLD activity score. Samples with scores $\geq 5$ were diagnosed with NASH (13). Immunohistochemical analyses were performed using antibodies against TNF- $\alpha$ and IL-6 (rabbit antibody; dilution 1:200) according to the protocol of our previous study (14).

Oil Red $O$ staining. For Oil Red O staining, frozen liver samples were cut into $5-\mu \mathrm{m}$-thick sections and subsequently air-dried onto slides. First, specimens were fixed in $4 \%$ paraformaldehyde, and washed with running tap water for 1-10 min. The specimens were then rinsed with $60 \%$ isopropanol, stained with freshly prepared Oil Red O working solution for $15 \mathrm{~min}$ and rinsed with $60 \%$ isopropanol. Finally, the nuclei were lightly stained by dipping the slides into hematoxylin solution five times and rinsing with distilled water for examination using light microscopy (magnification, x200).

Antioxidant assay. At the end of the experiment, the mice were anesthetized using chloral hydrate and sacrificed by cervical dislocation. Subsequently, the livers were quickly harvested and placed on ice. The antioxidant enzyme activities, including the activities of SOD and GSH-px, and the MDA content were determined, according to the protocol of our previous study (14).

Measurement of ROS of liver tissue. The ROS level of the liver tissue was detected using the redox-sensitive fluorescent dye DCFH-DA. Briefly, the frozen liver samples were cut into $5-\mu \mathrm{m}$-thick sections and left to air dry onto slides. First, the specimens were fixed in $4 \%$ paraformaldehyde and washed three times with PBS. The specimens were incubated with DCFH-DA $(10 \mu \mathrm{mol} / \mathrm{l})$, which was diluted with $\mathrm{PBS}$ at $37^{\circ} \mathrm{C}$ for $30 \mathrm{~min}$, and then washed five times with PBS. Images of the relative level of fluorescent product were captured using a fluorescence microscope connected to an imaging system (BX50-FLA; Olympus Corporation). ImageJ 1.47i software (National Institutes of Health) was used to analyze the mean fluorescence intensity of DCFH-DA, which indirectly detected the level of ROS.

Western blotting. The protein expression levels of TNF- $\alpha$, IL-6, $\mathrm{NF}-\kappa \mathrm{B}, \mathrm{p}-\mathrm{NF}-\mathrm{\kappa} \mathrm{B}$ and $\beta$-actin in the liver tissues obtained from rats in different groups were detected by western blot analysis. The liver tissues were incubated in lysis buffer supplied with a cocktail of phosphatase inhibitors (Roche Diagnostics, Indianapolis, IN, USA). Total protein was quantified using the bicinchoninic acid protein assay kit (Pierce; Thermo Fisher Scientific, Inc., Waltham, MA, USA). The protocol and semiquantitative analysis were 

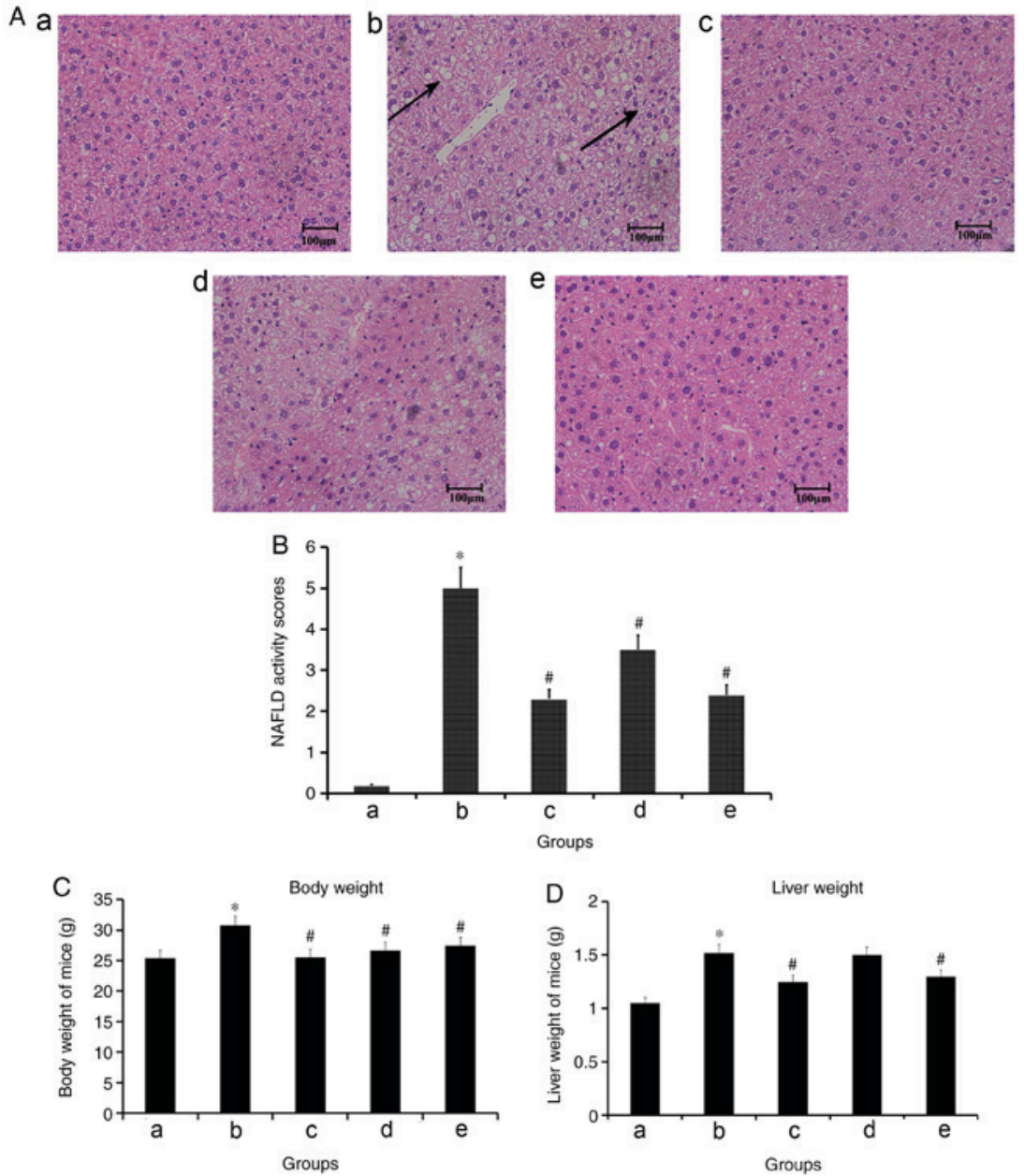

Figure 1. Evaluation of liver morphology and NAFLD activity scores. (A) Representative examples of hematoxylin and eosin-stained hepatic sections. (B) NAFLD activity scores of liver tissue. (C) Body weight and (D) liver weight of mice from the (a) control, (b) model, (c) 4 mg/kg/day simvastatin-treated, (d) $100 \mathrm{mg} / \mathrm{kg} /$ day TMP-treated and (e) $200 \mathrm{mg} / \mathrm{kg} /$ day TMP-treated groups are shown. Hepatic steatosis and inflammatory cell infiltration (black arrows) were observed in the model group. Magnification, x $200 .{ }^{*} \mathrm{P}<0.05$ compared with the control group; ${ }^{*} \mathrm{P}<0.05$ compared with the model group. NAFLD, non-alcoholic fatty liver disease; TMP, tetramethylpyrazine.

performed according the protocol of our previous study (14). The following antibodies were used: TNF- $\alpha, \mathrm{IL}-6, \mathrm{NF}-\kappa \mathrm{B}, \mathrm{p}-\mathrm{NF}-\kappa \mathrm{B}$ (rabbit antibody; dilution 1:1,000) and $\beta$-actin (rabbit antibody; dilution 1:1,000). The secondary antibodies used were the following: Horseradish peroxidase-conjugated anti-rabbit immunoglobulin G (IgG; cat. no. 7074; dilution 1:5,000; Santa Cruz Biotechnology, Inc.) or anti-mouse IgG (cat. no. 7076; dilution 1:5,000; Santa Cruz Biotechnology, Inc.).

Statistical analysis. SPSS version 13.0 software for Windows (SPSS, Inc., Chicago, IL, USA) was used for statistical analysis. All values are presented as the mean \pm standard deviation of the mean for the indicated number of measurements. For comparisons among multiple groups, one-way analysis of variance followed by the Least Significant Difference test was performed. $\mathrm{P}<0.05$ was considered to indicate a statistically significant difference.

\section{Results}

Effects of TMP treatment on liver injury in a high fat diet-induced model of NASH. The administration of a high fat diet to C57BL/6 mice resulted in a classical pathophysiological model of NASH, with microvesicular and macrovesicular steatosis, indicative of disturbed lipid metabolism, and few foci of inflammatory cell accumulations in the liver. Treatment with simvastatin and TMP decreased the size and number of macrovesicular steatosis and inflammation, compared with model mice (Fig. 1A). In addition, compared with the control group, the NAFLD activity scores of the model group were significantly increased. However, compared with the model group, the NAFLD activity scores of $4 \mathrm{mg} / \mathrm{kg} / \mathrm{day}$ simvastatin-treated, $100 \mathrm{mg} / \mathrm{kg} /$ day TMP-treated and $200 \mathrm{mg} / \mathrm{kg} /$ day TMP-treated groups were significantly decreased (Fig. 1B). Compared with the control group, the body weight and liver weight of mice in the model group were significantly increased. However, compared with the model group, the body weight and liver weight of mice in the simvastatin-treated and $200 \mathrm{mg} / \mathrm{kg} /$ day TMP-treated groups, and the body weight of mice in the $100 \mathrm{mg} / \mathrm{kg} /$ day TMP-treated group, were significantly decreased (Fig. 1C and D).

TMP does not decrease the levels of serum glucose, ALT, TG, $T C$ or $L D L-C$. It was demonstrated that, compared with the control group, the levels of blood glucose, ALT, TG, TC and LDL-C were increased in the model group. However, compared with the model group, 100 and $200 \mathrm{mg} / \mathrm{kg} /$ day TMP did not 
Table I. Effect of TMP on levels of SG, TG, TC, LDL-C and ALT ( $n=12)$.

\begin{tabular}{lcccccc}
\hline Group & Dose $(\mathrm{mg} / \mathrm{kg} / \mathrm{day})$ & $\mathrm{SG}(\mathrm{mmol} / \mathrm{l})$ & $\mathrm{TG}(\mathrm{mmol} / \mathrm{l})$ & $\mathrm{TC}(\mathrm{mmol} / \mathrm{l})$ & LDL-C $(\mathrm{mmol} / \mathrm{l})$ & ALT \\
\hline Control & - & $8.9 \pm 1.3$ & $0.54 \pm 0.07$ & $1.91 \pm 0.20$ & $0.20 \pm 0.05$ & $39.5 \pm 7.9$ \\
Model & - & $10.6 \pm 1.4^{\mathrm{a}}$ & $0.67 \pm 0.09^{\mathrm{a}}$ & $3.72 \pm 0.84^{\mathrm{a}}$ & $0.28 \pm 0.05^{\mathrm{a}}$ & $53.4 \pm 13.3^{\mathrm{a}}$ \\
Low-dose TMP & 100 & $10.4 \pm 1.6$ & $0.62 \pm 0.17$ & $3.82 \pm 0.99$ & $0.30 \pm 0.05$ & $47.3 \pm 6.6$ \\
High-dose TMP & 200 & $9.3 \pm 1.3$ & $0.58 \pm 0.04$ & $3.76 \pm 0.66$ & $0.27 \pm 0.05$ & $40.8 \pm 10.2^{\mathrm{b}}$ \\
Simvastatin & 4 & $9.5 \pm 1.7$ & $0.59 \pm 0.10$ & $2.77 \pm 0.64^{\mathrm{b}}$ & $0.29 \pm 0.05$ & $43.8 \pm 9.6$ \\
\hline
\end{tabular}

${ }^{\mathrm{a}} \mathrm{P}<0.05$, compared with control group; ${ }^{\mathrm{P}} \mathrm{P}<0.05$, compared with model group. TMP, tetramethylpyrazine; $\mathrm{SG}$, serum glucose; TG, triglyceride; TC, total cholesterol; LDL-C, low-density lipoprotein-cholesterol; ALT, alanine aminotransferase.
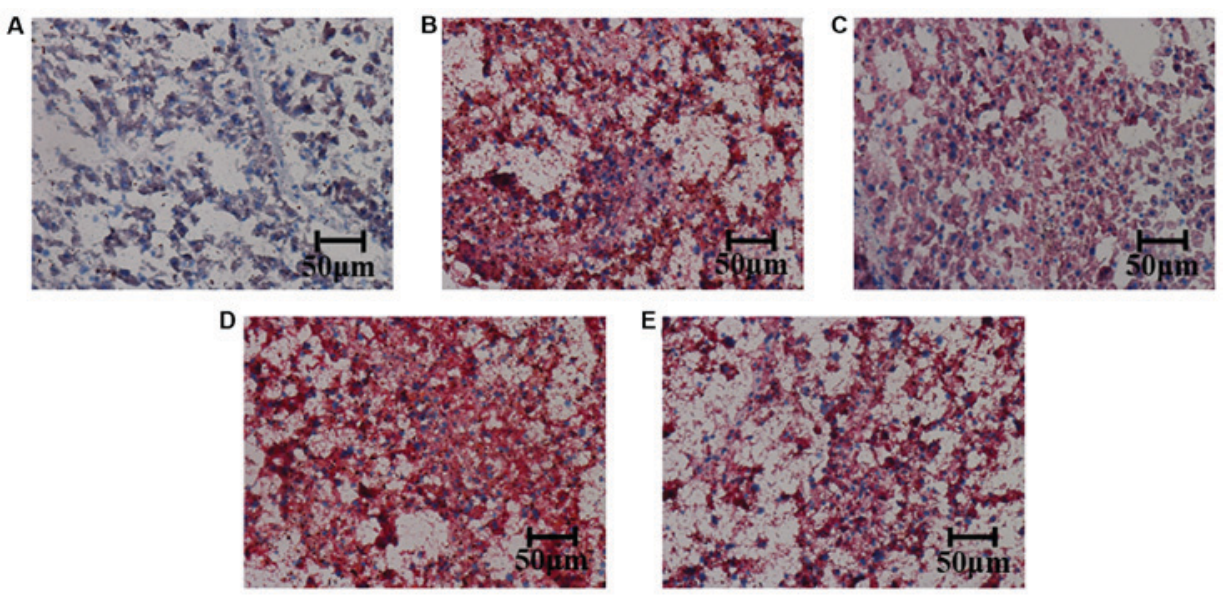

Figure 2. Evaluation of fat deposition in the liver tissue. Representative examples of Oil Red O staining hepatic sections from the (A) control, (B) model, (C) $4 \mathrm{mg} / \mathrm{kg} /$ day simvastatin-treated, (D) $100 \mathrm{mg} / \mathrm{kg} / \mathrm{day}$ TMP-treated and (E) $200 \mathrm{mg} / \mathrm{kg} /$ day TMP-treated groups are shown. Magnification, x200. TMP, tetramethylpyrazine.

decrease the levels of serum glucose, TC, TG and LDL-C, whereas the levels of ALT were decreased. Furthermore, compared with the model group, simvastatin did not decrease the levels of serum glucose, ALT, TG and LDL-C, whereas the levels of TC were decreased (Table I).

High doses of TMP can improve fat deposition in the liver. The fat deposition of the liver was detected by Oil Red O staining. It was observed that, compared with the control group, the fat deposition in the liver was increased in the model group. The fat deposition of liver was decreased in the simvastatin 4 and $200 \mathrm{mg} / \mathrm{kg} /$ day TMP-treated groups; however, not in the $100 \mathrm{mg} / \mathrm{kg} / \mathrm{day}$ TMP-treated group, compared with the model group (Fig. 2).

TMP improves the levels of MDA, SOD and GSH-px in liver tissues. Compared with the control group, the model group exhibited GSH-px and SOD levels that were 36.2 and $14.5 \%$ lower, respectively, in the liver tissues; however, the MDA level was $26.4 \%$ higher in the model group. It was identified that, compared with the model group, the C57BL/6J mice treated with 100 and $200 \mathrm{mg} / \mathrm{kg} /$ day TMP exhibited 24.9 and $55.0 \%$ higher GSH-px levels, respectively, in the liver tissues; however, the SOD levels were not increased. Additionally, the MDA level was 24.0 and $24.5 \%$ lower, respectively, in the 100 and $200 \mathrm{mg} / \mathrm{kg} /$ day TMP groups, compared

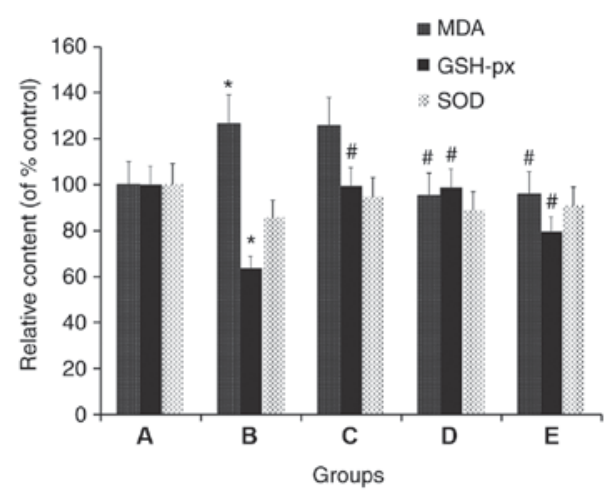

Figure 3. TMP improves the levels of MDA, SOD and GSH-px in the liver tissue. The levels of MDA, SOD and GSH-px in the (A) control, (B) model, (C) $4 \mathrm{mg} / \mathrm{kg} / \mathrm{day}$ simvastatin-treated, (D) $100 \mathrm{mg} / \mathrm{kg} / \mathrm{day}$ TMP-treated and (E) $200 \mathrm{mg} / \mathrm{kg} / \mathrm{day}$ TMP-treated groups are shown. $\mathrm{P}<0.05$ compared with the control group; ${ }^{\text {}} \mathrm{P}<0.05$ compared with the model group. TMP, tetramethylpyrazine; MDA, malondialdehyde; SOD, superoxide dismutase; GSH-pX, glutathione peroxidase.

with that in the model group. Furthermore, compared with model group, the C57BL $/ 6 \mathrm{~J}$ mice treated with $4 \mathrm{mg} / \mathrm{kg} / \mathrm{day}$ simvastatin had 55.9 and $10.5 \%$ higher GSH-px and SOD levels, respectively, in the liver tissues, whereas the MDA levels did not increase (Fig. 3). 

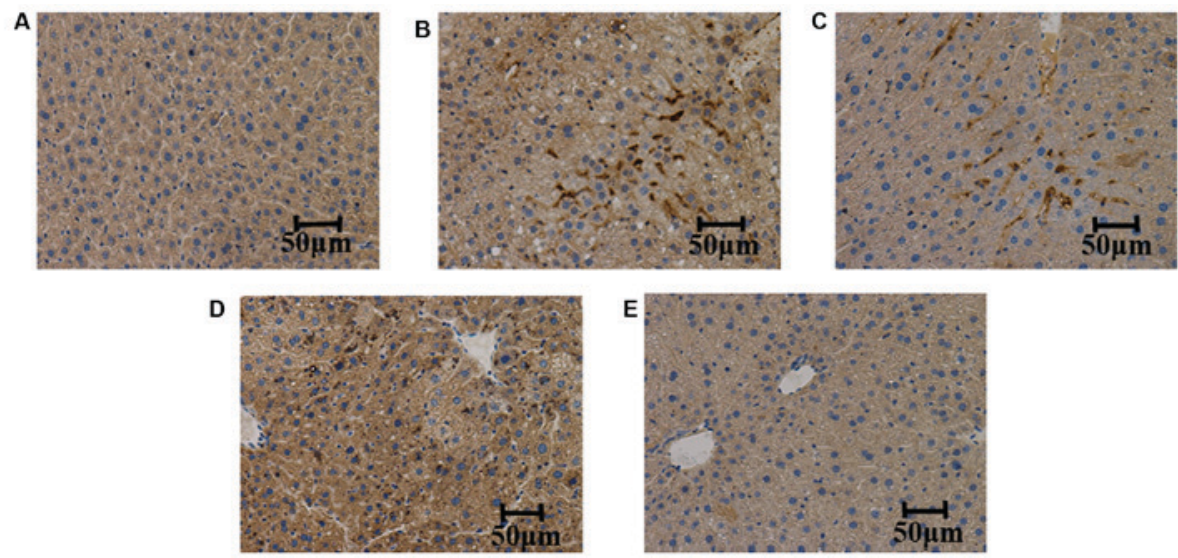

Figure 4. Effects of TMP on the expression of TNF- $\alpha$ in the liver tissues of mice fed a high fat diet. Immunohistochemical staining of representative liver tissue sections from the (A) control, (B) model, (C) $4 \mathrm{mg} / \mathrm{kg} /$ day simvastatin-treated, (D) $100 \mathrm{mg} / \mathrm{kg} / \mathrm{day}$ TMP-treated and (E) $200 \mathrm{mg} / \mathrm{kg} / \mathrm{day} \mathrm{TMP}$-treated groups are shown. Magnification, x200. Positive (brown) staining indicates the expression of TNF- $\alpha$. TMP, tetramethylpyrazine; TNF- $\alpha$, tumor necrosis factor- $\alpha$.
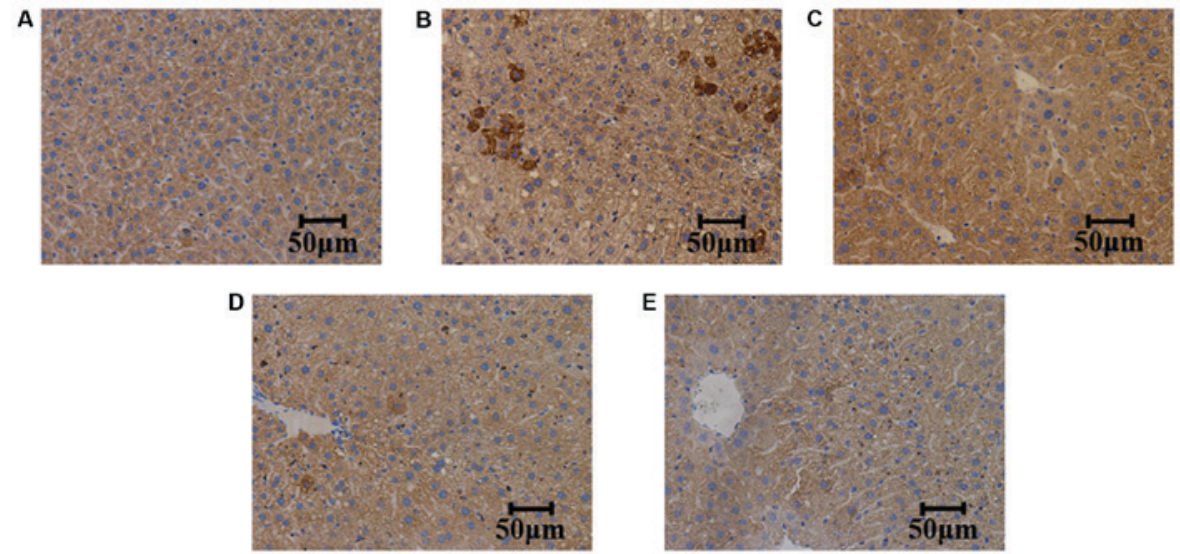

Figure 5. Effects of TMP on the expression of IL-6 in the liver tissues of mice fed a high fat diet. Immunohistochemical staining of representative liver tissue sections from the (A) control, (B) model, (C) $4 \mathrm{mg} / \mathrm{kg} /$ day simvastatin-treated, (D) $100 \mathrm{mg} / \mathrm{kg} / \mathrm{day}$ TMP-treated and (E) $200 \mathrm{mg} / \mathrm{kg} / \mathrm{day} \mathrm{TMP}$-treated groups are shown. Magnification, x200. Positive (brown) staining indicates the expression of IL-6. TMP, tetramethylpyrazine; IL-6, interleukin-6.

TMP suppresses the expression of TNF- $\alpha$ and IL- 6 in liver tissues. The expression levels of TNF- $\alpha$ and IL-6 were measured in the liver tissues of the mice. It was observed that, compared with the control group, the expression levels of TNF- $\alpha$ and IL- 6 were increased in the model group. Furthermore, compared with the model group, TMP (100 and $200 \mathrm{mg} / \mathrm{kg} / \mathrm{day}$ ) treatment reduced the expression levels of TNF- $\alpha$ and IL-6; however, simvastatin did not suppress the expression of TNF- $\alpha$ and IL-6, as determined by immunohistochemistry (Figs. 4 and 5).

TMP suppresses the expression of TNF- $\alpha, I L-6$ and NF- $\kappa B$, and the phosphorylation of $N F-\kappa B$ in liver tissues. Western blot analysis was conducted to determine the expression of TNF- $\alpha$, IL- 6 and NF- $\kappa B$, and the phosphorylation of NF- $-\mathrm{B}$ in the liver tissues. It was demonstrated that, compared with the control group, the expression levels of TNF- $\alpha$ and IL-6, and the ratio of $\mathrm{p}-\mathrm{NF}-\mathrm{\kappa B} / \mathrm{NF}-\mathrm{\kappa B}$ were increased in the model group. Compared with the model group, TMP (100 and $200 \mathrm{mg} / \mathrm{kg} / \mathrm{day})$ and simvastatin treatment reduced the expression levels of TNF- $\alpha$ and IL- 6 , and the ratio of p-NF- $\kappa B / N F-\kappa B$ (Fig. 6).

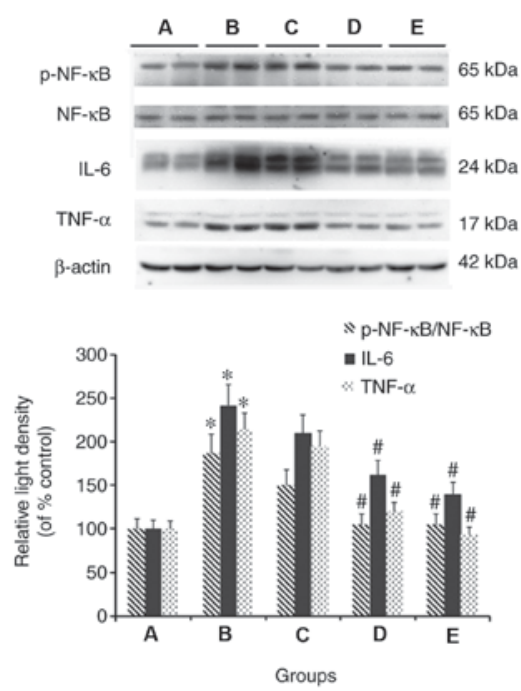

Figure 6. Effects of TMP on the expression of TNF- $\alpha$, IL- 6 and the ratio of p-NF- $\mathrm{kB} / \mathrm{NF}-\kappa \mathrm{B}$ in the liver tissues of mice fed a high fat diet. Western blot analysis of representative liver tissue samples from the (A) control, (B) model, (C) $4 \mathrm{mg} / \mathrm{kg} / \mathrm{day}$ simvastatin-treated, (D) $100 \mathrm{mg} / \mathrm{kg} /$ day TMP-treated and (E) $200 \mathrm{mg} / \mathrm{kg} /$ day TMP-treated groups are shown. "P<0.05, compared with the control group; ${ }^{*} \mathrm{P}<0.05$, compared with the model group. $T M P$, tetramethylpyrazine; TNF- $\alpha$, tumor necrosis factor- $\alpha$; IL-6, interleukin- 6 ; NF- $\mathrm{kB}$, nuclear factor $\kappa B$. 

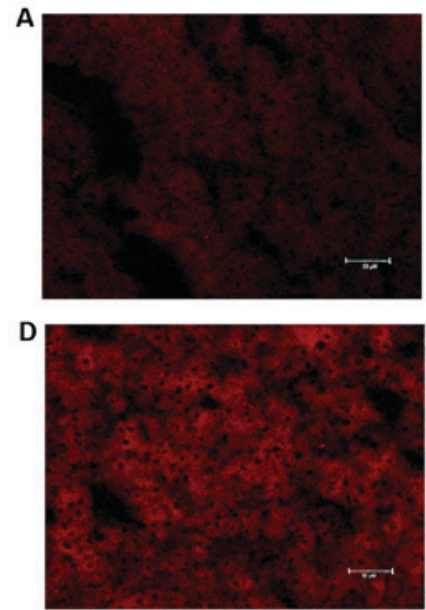
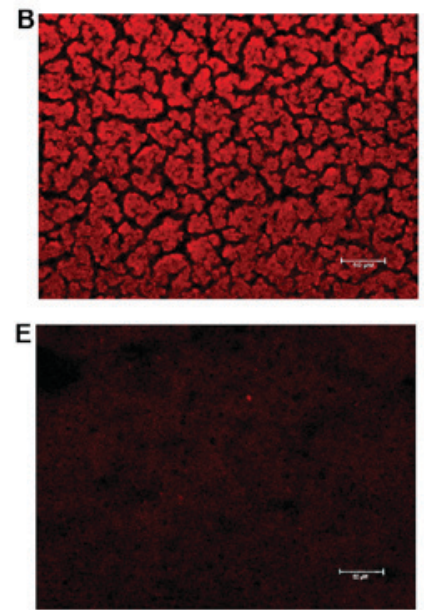
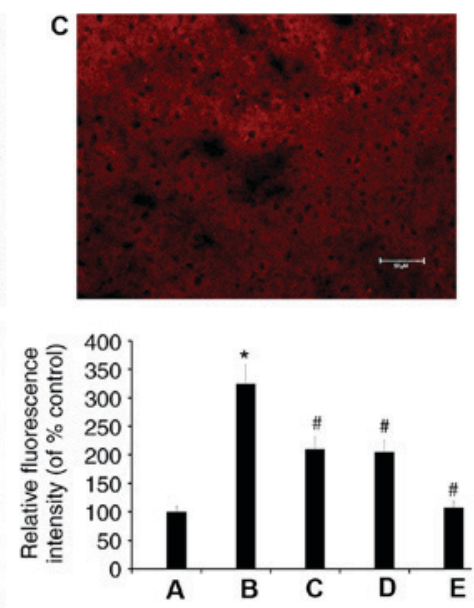

Figure 7. Effects of TMP on ROS level of the liver tissues. Fluorescence intensity of representative liver tissue sections from the (A) control, (B) model, (C) $4 \mathrm{mg} / \mathrm{kg}$ /day simvastatin-treated, (D) $100 \mathrm{mg} / \mathrm{kg} /$ day TMP-treated and (E) $200 \mathrm{mg} / \mathrm{kg} /$ day TMP-treated groups are shown. Magnification, x200. *P<0.05, compared with the control group; ${ }^{\#} \mathrm{P}<0.05$, compared with the model group. TMP, tetramethylpyrazine; ROS, reactive oxygen species.

TMP suppresses the production of ROS induced by a high fat diet. It was found that, compared with the control group, the production of ROS was increased in the livers of C57BL/6 mice fed a high fat diet. It was also demonstrated that, compared with the model group, TMP (100 and $200 \mathrm{mg} / \mathrm{kg} / \mathrm{day})$ and simvastatin treatment reduced the production of ROS (Fig. 7).

\section{Discussion}

NAFL is characterized by an accumulation of hepatocellular lipids, mainly triglycerides, whereas NASH is characterized by the presence of hepatocellular damage and inflammation (15), which can drive the development of liver fibrosis (16), the strongest predictor of NAFLD-associated mortality (17). If inflammation is inhibited, the progression of NAFL to NASH may be delayed or repressed, which may also restrict the development of liver fibrosis. The present study investigated the effect of TMP on liver inflammation induced by a high fat diet. At 8 weeks, blood was collected through the eyeball of a random selection of $\sim 10 \%$ of mice fed a high fat diet (six mice) and $50 \%$ of mice fed a regular diet (six mice), and the levels of AST and ALT were detected. The results showed that, compared with mice fed a regular diet, the levels of AST and ALT were increased (data not shown) in the mice fed a high fat diet. According to this, it was possible to confirm the presence of liver damage in the mice fed a high fat diet. Therefore, inflammation of the liver was not examined at 8 weeks, but at 12 weeks in the mice fed a high fat diet. The murine NAFLD model was established by feeding mice a high fat diet for 8 weeks. NAFL induced in $\mathrm{C} 57 \mathrm{BL} / \mathrm{J}$ mice imitates human NAFLD, and the standard for the establishment of the murine model for NAFLD is the NAFLD activity score. Therefore, the NAFLD activity score was considered to be suitable for the murine NAFLD model. Others have also used the NAFLD activity score to examine the liver of C57BL/6JRj mice (18). In the present study, if there was no intervention of the model group, NAFL progressed to NASH and the NAFLD activity scores of the model group were $>5$. It was observed that simvastatin and $200 \mathrm{mg} / \mathrm{kg} /$ day TMP lowered the increased body weight and liver weight of the mice, which had been induced by a high fat diet. It was hypothesized that the loss of weight following treatment with simvastatin and $200 \mathrm{mg} / \mathrm{kg} /$ day TMP is due to the reduction in the weight of the liver. However, $100 \mathrm{mg} / \mathrm{kg} /$ day TMP only lowered the body weight of the mice. According to the manufacturer's instructions, the minimum lethal dose of TMP in mice is $700-800 \mathrm{mg} / \mathrm{kg}$ via intraperitoneal administration. Therefore, it was hypothesized that TMP also had the effect of weight loss. The effect of different doses of TMP on the body weight of mice requires further investigation.

In the present study, it was observed that TMP had no effect on serum glucose, TC, TG or LDL-C. However, TMP protected hepatocytes from damage, as it was demonstrated that $200 \mathrm{mg} / \mathrm{kg} /$ day TMP decreased the ALT level. The positive control agent (4 mg/kg/day simvastatin) did not decrease the serum levels of glucose, ALT, TG or LDL-C; however, it decreased the TC level. Therefore, compared with simvastatin, TMP had a protective effect on the liver. Future experiments aim to investigate the molecular mechanism of TMP in preventing NASH. The pathogenesis of NASH includes insulin resistance, increased inflammation (TNF- $\alpha$ and IL-6) and increased oxidative damage (18). The levels of TNF- $\alpha$ and other TNF-induced cytokines, including IL-6 and IL-8, were higher in animals with NASH. This is likely associated with the progression of NASH in liver cirrhosis (19). In the present study, it was observed that TMP inhibited the liver inflammation induced by a high fat diet. The expression levels of TNF- $\alpha$ and IL-6 were increased in C57BL/J mice fed a high fat diet. Compared with the model group, TMP decreased the expression of TNF- $\alpha$ and IL-6. However, the positive control agent, simvastatin, at a dose of $4 \mathrm{mg} / \mathrm{kg} /$ day did not have this effect. Therefore, it can be deduced that TMP, rather than simvastatin, can inhibit liver inflammation. It was previously reported that TNF- $\alpha$ can activate NF- $\kappa \mathrm{B}$ (20). In the present study, it was also observed that the phosphorylation of $\mathrm{NF}-\kappa \mathrm{B}$ was increased in C57BL/J mice fed a high fat diet, and TMP inhibited the increased phosphorylation of $\mathrm{NF}-\kappa \mathrm{B}$. Therefore, TMP can inhibit the inflammatory response in the liver induced by a high fat diet by inhibiting the expression of TNF- $\alpha$ and the phosphorylation of NF- $\mathrm{NB}$. 
It was previously reported that TNF- $\alpha$ is a regulator of the generation of $\operatorname{ROS}(21,22)$. ROS are essential for normal physiological functions, gene expression, cell growth, defense against infections and the control of vascular endothelial cells (23). However, when ROS production exceeds scavenging abilities, cells are exposed to oxidative stress and are damaged (24,25). The present study investigated whether ROS levels were increased in the livers of mice fed a high fat diet following an increase of TNF- $\alpha$, and whether TMP inhibits the increase of ROS. The results demonstrated that the levels of ROS and TNF- $\alpha$ were increased in the livers of mice fed a high fat diet. TMP treatment lowered the increased levels of ROS and TNF- $\alpha$ induced by the high fat diet. It can be deduced that TMP protects the liver by inhibiting the TNF- $\alpha / \mathrm{ROS} / \mathrm{NF}-\kappa \mathrm{B}$ signaling pathway. The effect of simvastatin on ROS requires further investigation.

In conclusion, the present study is the first to demonstrate, to the best of our knowledge, that TMP inhibits the inflammatory response of the liver by reducing the production of TNF- $\alpha$ and IL-6, the generation of ROS and subsequently NF- $\kappa \mathrm{B}$ activation. TMP has the potential to become an effective therapeutic drug to treat liver disease by inhibiting the inflammatory response. A limitation of the present study is that time course data on the effect of TMP were absent. Further investigations are required to investigate the effect of TMP on a high fat-induced murine NAFLD model in time-dependent manner. Although it was observed that TMP suppressed the production of TNF- $\alpha$ and IL-6 in vivo, for a definitive conclusion, an in vitro investigation of TMP is required.

\section{Acknowledgements}

Not applicable.

\section{Funding}

This study was supported by grants from the National Natural Science Foundation of China (grant no. 81671391) and Beijing Hospital Nova Project (grant no. BJ-2016-033).

\section{Availability of data and materials}

The datasets used and/or analyzed during the current study are available from the corresponding author on reasonable request.

\section{Authors' contributions}

$\mathrm{BC}$ and $\mathrm{YM}$ performed the experiments and analyzed the data. $\mathrm{XX}$ and JW contributed to the conception and design of the study. GH and YL designed the present study and revised the manuscript. All authors read and approved the final manuscript.

\section{Ethics approval and consent to participate}

The study was approved by The Research Ethics Committee of the China Academy of Chinese Medical Sciences (Beijing, China), in accordance with the National Institutes of Health Guidelines for the Care and Use of Laboratory Animals (National Institutes of Health, Bethesda, MD, USA). All animals were treated in accordance with the guidelines and regulations for the use and care of animals of the Center for Laboratory Animal Care, China Academy of Chinese Medical Sciences.

\section{Patient consent for publication}

Not applicable.

\section{Competing interests}

The authors declare that they have no competing interests.

\section{References}

1. Ghaemi A, Hosseini N, Osati S, Naghizadeh MM, Dehghan A, Ehrampoush E, Honarvar B and Homayounfar R: Waist circumference is a mediator of dietary pattern in non-alcoholic fatty liver disease. Sci Rep 8: 4788, 2018.

2. Komazaki R, Katagiri S, Takahashi H, Maekawa S, Shiba T, Takeuchi Y, Kitajima Y, Ohtsu A, Udagawa S, Sasaki N, et al: Periodontal pathogenic bacteria, Aggregatibacter actinomycetemcomitans affect non-alcoholic fatty liver disease by altering gut microbiota and glucose metabolism. Sci Rep 7: 13950, 2017.

3. Torres DM, Williams CD and Harrison SA: Features, diagnosis, and treatment of nonalcoholic fatty liver disease. Clin Gastroenterol Hepatol 10: 837-858, 2012.

4. Schröder T, Kucharczyk D, Bär F, Pagel R, Derer S, Jendrek ST, Sünderhauf A, Brethack AK, Hirose M, Möller S, et al: Mitochondrial gene polymorphisms alter hepatic cellular energy metabolism and aggravate diet-induced non-alcoholic steatohepatitis. Mol Metab 5: 283-295, 2016.

5. Schuppan D and Schattenberg JM: Non-alcoholic steatohepatitis: Pathogenesis and novel therapeutic approaches. J Gastroenterol Hepatol 28 (Suppl 1): S68-S76, 2013.

6. Ekstedt M, Franzén LE, Mathiesen UL, Thorelius L, Holmqvist M, Bodemar G and Kechagias S: Long-term follow-up of patients with NAFLD and elevated liver enzymes. Hepatology 44: 865-873, 2006.

7. Schattenberg JM and Schuppan D: Nonalcoholic steatohepatitis: The therapeutic challenge of a global epidemic. Curr Opin Lipidol 22: 479-488, 2011.

8. Yeom GG, Min S and Kim SY: 2,3,5,6-Tetramethylpyrazine of Ephedra sinica regulates melanogenesis and inflammation in a UVA-induced melanoma/keratinocytes co-culture system. Int Immunopharmacol 18: 262-269, 2014.

9. Ran X, Ma L, Peng C, Zhang H and Qin LP: Ligusticum chuanxiong Hort: A review of chemistry and pharmacology. Pharm Biol 49: 1180-1189, 2011.

10. Shao Z, Wang L, Liu S and Wang X: Tetramethylpyrazine protects neurons from oxygen-glucose deprivation-induced death. Med Sci Monit 23: 5277-5282, 2017.

11. Gong X, Ivanov VN, Davidson MM and Hei TK: Tetramethylpyrazine (TMP) protects against sodium arsenite-induced nephrotoxicity by suppressing ROS production, mitochondrial dysfunction, pro-inflammatory signaling pathways and programed cell death. Arch Toxico 89: 1057-1070, 2015.

12. Kleiner DE, Brunt EM, Van Natta M, Behling C, Contos MJ, Cummings OW, Ferrell LD, Liu YC, Torbenson MS Unalp-Arida A, et al: Design and validation of a histological scoring system for nonalcoholic fatty liver disease. Hepatology 41 : 1313-1321, 2005.

13. Staroń R, Van Swelm RP, Lipiński P, Gajowiak A, Lenartowicz M, Bednarz A,GajewskaM,Pieszka M,Laarakkers CM,Swinkels DW and Starzyński RR: Urinary hepcidin levels in iron-deficient and iron-supplemented piglets correlate with hepcidin hepatic mRNA and serum levels and with body iron status. PLoS One 10: e136695, 2015.

14. Wei J, Zhen YZ, Cui J, He FL, Shen T, Hu G, Ren XH and Lin YJ: Rhein lysinate decreases inflammation and adipose infiltration in $\mathrm{KK} / \mathrm{H} 1 \mathrm{~J}$ diabetic mice with non-alcoholic fatty liver disease. Arch Pharm Res. 39: 960-969, 2016.

15. Rinella ME: Nonalcoholic fatty liver disease: A systematic review. JAMA 313: 2263-2273, 2015.

16. Fujii $\mathrm{H}$ and Kawada N: Inflammation and fibrogenesis in steatohepatitis. J Gastroenterol 47: 215-225, 2012. 
17. Morrison MC, Kleemann R, van Koppen A, Hanemaaijer R and Verschuren L: Key inflammatory processes in human NASH are reflected in $\mathrm{Ldlr}^{--}$.Leiden mice: A translational gene profiling study. Front Physiol 9: 132, 2018.

18. Henkel J, Alfine E, Saín J, Jöhrens K, Weber D, Castro JP, König J, Stuhlmann C, Vahrenbrink M, Jonas W, et al: Soybean oil-derived poly-unsaturated fatty acids enhance liver damage in NAFLD induced by dietary cholesterol. Nutrients 10: pii: E1326, 2018.

19. Hebbard L and George J: Animal models of nonalcoholic fatty liver disease. Nat Rev Gastroenterol Hepatol 8: 35-44, 2011.

20. Hayden MS and Ghosh S: Regulation of NF- $\kappa$ B by TNF family cytokines. Semin Immunol 26: 253-266, 2014.

21. Abd El-Kader SM, Al-Shreef FM and Al-Jiffri OH: Biochemical parameters response to weight loss in patients with non-alcoholic steatohepatitis. Afr Health Sci 16: 242-249, 2016.

22. Kastl L, Sauer SW, Ruppert T, Beissbarth T, Becker MS, Süss D, Krammer PH, and Gülow K: TNF- $\alpha$ mediates mitochondrial uncoupling and enhances ROS-dependent cell migration via NF- $\kappa \mathrm{B}$ activation in liver cells. FEBS Lett 588: 175-183, 2014.
23. Blaser H, Dostert C, Mak TW and Brenner D: TNF and ROS crosstalk in inflammation. Trends Cell Biol 26: 249-261, 2016.

24. Montezano AC and Touyz RM: Reactive oxygen species and endothelial function-role of nitric oxide synthase uncoupling and Nox family nicotinamide adenine dinucleotide phosphate oxidases. Basic Clin Pharmacol Toxicol 110: 87-94, 2012.

25. Togo M, Konari N, Tsukamoto M, Kimoto R, Yamaguchi T, Takeda $\mathrm{H}$ and Kambayashi I: Effects of a high-fat diet on superoxide anion generation and membrane fluidity in liver mitochondria in rats. J Int Soc Sports Nutr 15: 13, 2018.

c) (i) (9) This work is licensed under a Creative Commons

EY No ND Attribution-NonCommercial-NoDerivatives 4.0 International (CC BY-NC-ND 4.0) License. 